\title{
Design and Simulation Analysis of Switch Gear Live Detection Robot
}

\author{
Yingjie $\mathrm{Lu}^{\mathrm{a}, 1}$, Chao Huang ${ }^{\mathrm{a}}$, Miao $\mathrm{Su}^{\mathrm{a}}$, Miaolong $\mathrm{Hu}^{\mathrm{b}}$ \\ ${ }^{a}$ State Grid Ningxia Electric Power Maintenance Company, Yinchuan 750011, China \\ ${ }^{\mathrm{b}}$ Zhejiang Weisi Wireless Network Technology Co. LTD
}

\begin{abstract}
Aiming at the problems of low efficiency, low accuracy and long maintenance cycle of traditional manual detection methods for switch gear detection, robot is proposed to intelligently detect. Through the analysis on sizes of switch gear and switch room in electric power system, switch gear live detection robot is designed to automatically complete switch gear's real time live detection. While using D-H parameter method to describe mechanical arm, switch gear live detection robot's simulations are carried out by MATLAB, which analyse the spatial location of mechanical arm. Designed detection robot can ensure high efficiency and reduce manpower input.
\end{abstract}

Keywords. Switch gear; maintenance robot; lifting unit; MDOF manipulator

\section{Introduction}

High-voltage (HV) switch gear is a very important electrical equipment in power system, which is mainly used for power generation, transmission, distribution, power conversion and on-off, control and protection in consumption [1]. Insulation deterioration often occurs in the long-term operation of electrical equipment for the influence of working environment factors such as electricity, heat, chemistry and self-quality problems, which leads to partial discharge (PD) and equipment failure. Its reliability is related to power quality and reliability of power supply in power system [2,3]. Periodic manual overhaul is often used to detect partial discharge in HV switch gear at present. This method has a long overhaul period and cannot find the defects in time between the two overhauls. It may also overhaul the faultless equipment, resulting in resource waste and cost increase $[4,5]$. This mode is not conducive to the long-term and stable operation of power system, nor to the condition maintenance of HV switch gear equipment. In addition, professional training on inspection technology and safety is required in the early period of manual overhaul. Lighting and ventilation equipment should be switched off to prevent interference during inspection. The detection environment is harsh and the overhaul cost is high.

Special detection sensors on the market use the detection of transient ground voltage signal as the design principle: the magnitude of transient ground voltage (also called ground wave) signal is related to the severity of partial discharge and the position of

\footnotetext{
${ }^{1}$ Corresponding Author, Yingjie Lu, No. 1, Jinfeng Road, Jinfeng District, Yinchuan City, Ningxia Hui Autonomous Region, China, State Grid Ningxia Electric Power Maintenance Company; Email:346148630@qq.com.
} 
discharge point [6]. It is often necessary to detect several transient ground voltages in order to check a switch gear. The task of detection is heavy and the accuracy of detection position of switch gear is high, which is difficult to achieve manually. A live detection robot for switch gear is proposed to research the intelligence and specificity of detection robot, promote the practicability of detection robot and realize high-efficiency and highaccuracy switch gear detection.

\section{Design Overview}

The overall structure size of the inspection robot is mainly designed according to the size of the switch room and switch gear. Figure 1 shows the size of the switch gear in the power system. The final design of the switch gear live inspection robot needs to travel on the front and rear walkways as well as turn through the left and right-side walkways.

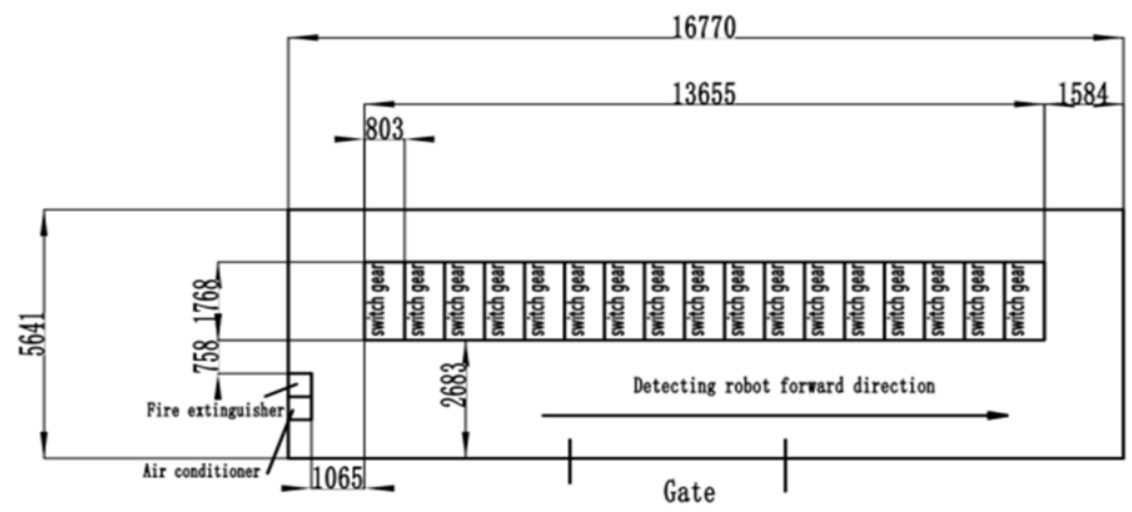

Figure 1. Dimension of switch gear in power system $(\mathrm{mm})$

The designed switch gear live detection robot needs to meet the following requirements:

1.The designed robot can carry out independent patrol inspection in the narrow switch-room space according to the predetermined route;

2.Design lifting platform to meet cabinet inspection requirements at different levels;

3.The multi-degree-of-freedom (MDOF) manipulator is designed to detect switch gear cabinets of different heights and different space positions in the same switch gear, and to position accurately and repeatedly.

As shown in Figure 2, the switch gear live detection robot consists of AGV, lifting unit, MDOF manipulator and electrical components. Sensors such as ground wave detection, ultrasonic partial discharge detection and switch gear keys are carried at the end of MDOF manipulator.

The overall size of the switch gear live detection robot is designed as $762 \mathrm{~mm} \times$ $510 \mathrm{~mm} \times 1611 \mathrm{~mm}$ according to the size of switch gear in Figure 1 and the general size data of switch gear (height $1.8 \mathrm{~m} \sim 2.0 \mathrm{~m}$, length about $800 \mathrm{~mm}$ ), so that each position of the detection robot moves corresponds to the position of a switch gear, and the robot can move freely and easily in the switch room. The MDOF manipulator is designed with an $X$ direction travel of $\mathrm{Sx}=150 \mathrm{~mm}$, and $\mathrm{Z}$ direction travel of $\mathrm{Sz}=917 \mathrm{~mm}$. The $\mathrm{Z}$-direction reachable height range is $\mathrm{Hz}=0 \sim \mathrm{Szmax}=0 \sim 2049 \mathrm{~mm}$.

$$
\mathrm{S}_{\mathrm{zmax}}=\mathrm{H}_{1}+\mathrm{S}_{\mathrm{Z}}+\mathrm{L}
$$




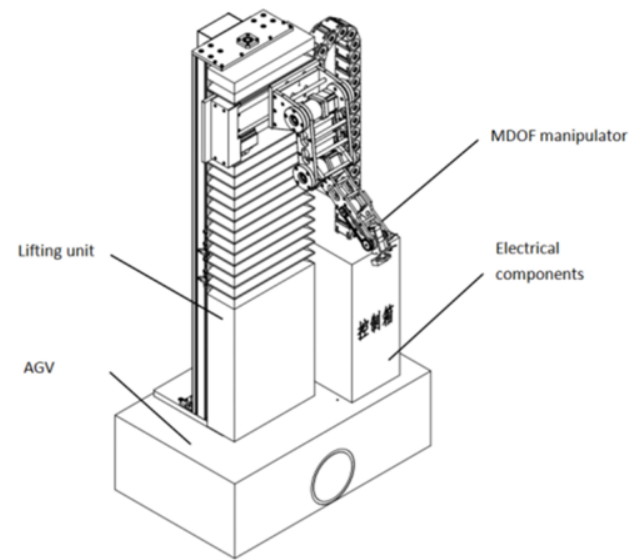

Figure 2. Structure composition of switch gear live detection mobile robot

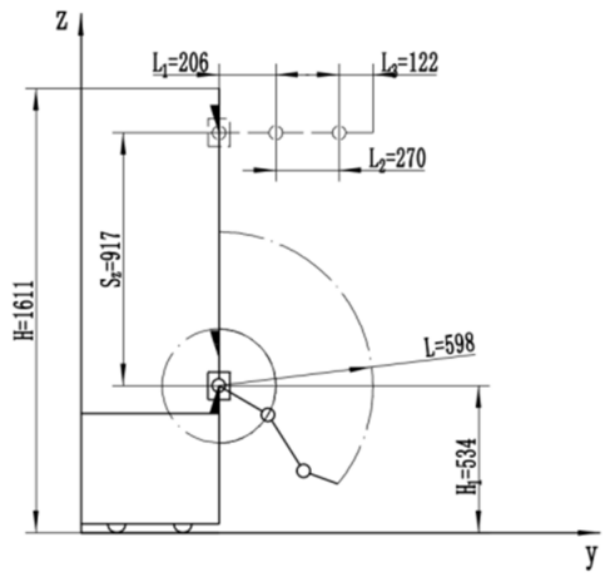

Figure 3. Inspection of robot (mm)

According to the modular structure design, the structure of switch gear live detection robot is divided into three modules: AGV, lifting unit and MDOF manipulator: $\mathrm{AGV}$ is used for omni-directional mobile independent patrol inspection, lifting unit adapts to switch gear cabinet layers of different heights, and MDOF manipulator is equipped to meet the full-degree scanning inspection in the cabinet. Custom AGV Zhongzhi-312 is selected to realize flexible movement in the narrow space of the switch room. Its parameters are as follows:

Table 1. AGV trolley parameters

\begin{tabular}{ccc}
\hline Maximum speed & Total weight of equipment & Drive \\
\hline $0.7 \mathrm{~m} / \mathrm{s}$ & $100 \mathrm{~kg}$ & $300 \mathrm{~N}$ \\
\hline
\end{tabular}

The designed live detection robot for switch gear works according to the following process:

1. The robot will reach the designated position in turn according to the predetermined route under the navigation of the AGV car. During the movement of the robot, the environment of the switch room can be monitored by video. 
2. Video acquisition and image processing are carried out on the high-voltage switch gear by the stereo camera carried at the end of the MDOF manipulator, and the points to be detected on the switch gear and the gap of the switch gear are identified and positioned by the image recognition algorithm.

3. According to the data of image recognition and positioning, control the MDOF manipulator to carry the corresponding detection tools, carry out the detection of ground wave, partial discharge, etc. at the corresponding detection points of the switch gear, and upload the data analysis to the remote monitoring terminal for inspection.

4. After the action is executed, the manipulator retracts to its initial position and the robot enters the next working position.

\section{Structural Design}

\subsection{Lifting unit}

The mobile robots in this inspection need to be equipped with lifting units for meeting the inspection requirements of switch cabinets at different levels.

As shown in Figure 4, linear motion unit is composed of guide screw, ball linear guide (with slider), supporting aluminum alloy profile and sealing system. Linear motion unit is widely used in various kinds of manipulators, which makes the mechanism compact and transmission smoother. Its friction coefficient is small, crawling is not easy to occur and the motion accuracy is higher [7, 8]. Sliding platform of the uni-axle robot is driven by rising lifting unit assisted by the sliding block on the straight guide rail. In addition, the groove sensor is combined with an inductive plate on the sliding platform for height positioning of the sliding platform.

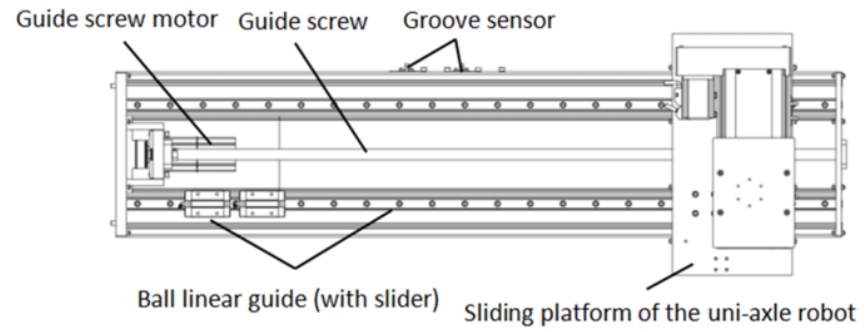

Figure 4. Lift unit drive

The weight of lifting unit, lifting unit driven by ball screw except for aluminum alloy profile and arm are $\mathrm{G}_{1}=29.31 \mathrm{~kg}, \mathrm{G}_{11}=10.94 \mathrm{~kg}$ and $\mathrm{G}_{2}=5.68 \mathrm{~kg}$ respectively. Operating speed is set to $\mathrm{v}=0.12 \mathrm{~m} / \mathrm{s}$, while the lead $\mathrm{P}_{\mathrm{B}}$ of the screw is calculated according to equation (2).

$$
\mathrm{P}_{\mathrm{B}}=\frac{\mathrm{v}}{\mathrm{n} \times \mathrm{i}}
$$

$\mathrm{i}$ is the ratio of the motor to the screw, where $\mathrm{i}=1 ; \mathrm{n}$ is the rated speed of the motor. Motor 60A3A02030 is selected, whose parameters are shown in Table 2: 
Table 2. Motor parameters

\begin{tabular}{cccc}
\hline Rated power & Rated speed & Rated voltage & Rated Torque \\
\hline $200 \mathrm{w}$ & $3000 \mathrm{RPM}$ & $24 \mathrm{~V}$ & $0.637 \mathrm{~N} \cdot \mathrm{m}$ \\
\hline
\end{tabular}

The lead $P_{B}=2.4 \mathrm{~mm}$ is calculated. So $P_{B}=5 \mathrm{~mm}$ is used when selecting the ball screw length $L_{B}=1300 \mathrm{~mm}$ according to the overall size design. The thread diameter $d$ of the screw is calculated according to equation (3).

$$
\mathrm{d}=\mathrm{a} \sqrt{\frac{\mu_{0} \mathrm{WL}}{\delta_{\mathrm{m}}}}
$$

a-bracing mode factor, when fixed at both ends $a=0.039 ; \mu_{0}$-static friction coefficient of guide rail, take $\mu_{0}=0.1 ; \mathrm{W}$-quality of towed workbench, i.e., $\mathrm{W}=\mathrm{G}_{11}+\mathrm{G}_{2}=16.62 \mathrm{~kg}$; Ldistance between bearing pivots, $\mathrm{L}=1.1 \times$ stroke $+(10 \sim 14) \times \mathrm{P}_{\mathrm{B}}=1068.7 \mathrm{~mm} ; \delta_{\mathrm{m}}$-ball screw maximum allowable axial deformation, take $\delta_{\mathrm{m}}=10 \mu \mathrm{m}$;

Screw LCS02-20-5-L1300-F20-P12 is selected finally combining calculated screw thread diameter $\mathrm{d}=16.436 \mathrm{~mm}$, selected screw shaft diameter $\mathrm{D}_{\mathrm{B}}=20 \mathrm{~mm}$ with with other dimensions.

Calibrate the torque of the nuclear power plant (60A3A02030) $\left(\mathrm{T}_{\mathrm{n}}=0.637\right.$ N.m) according to the calculation formula of ball screw thrust [9].

$$
\mathrm{P}=2 \pi \eta \mathrm{T} / \mathrm{P}_{\mathrm{B}}
$$

P-screw thrust, $\mathrm{P} \geq(10.94+5.68) \times 9.8=162.88 \mathrm{~N} ; \mathrm{P}_{\mathrm{B}}$-lead screw, $\mathrm{P}_{\mathrm{B}}=12 \mathrm{~mm}=0.012 \mathrm{~m} ; \mathrm{T}$ torque; $\eta$-screw drive efficiency, $\eta=0.9 \sim 0.95$;

$T=P P_{B} /(2 \pi \eta)=0.33 \sim 0.35 N \cdot m<T_{n}$, motor torque meets ball screw thrust requirements.

Lifting unit is used in mobile detection robot because of its unique advantages. Its external organ protective cover protects the internal drive mechanism, which can not only protect the mechanism from dust to extend its life, but also expand and deform to increase the appearance of the robot. The overall structure of lifting unit is simple and compact, which is easy to build by assembling various standard parts. Simple and convenient parts replacement can be realized in the process of use, which has strong interchangeability and thus the economy of the robot is increased.

\subsection{MDOF manipulator}

The MDOF manipulator is applied to the live detection robot of the switch gear, which can realize the full-range detection in the switch gear at a certain height. The electromechanical integrated modular joint structure is adopted, while motor driver, servo controller, motor and drive mechanism are integrated into one joint as shown in Figure 5. Compared with the separated structure, this structure has the advantages of short design cycle, convenient fabrication and simple maintenance in the later stage $[10,11]$. 


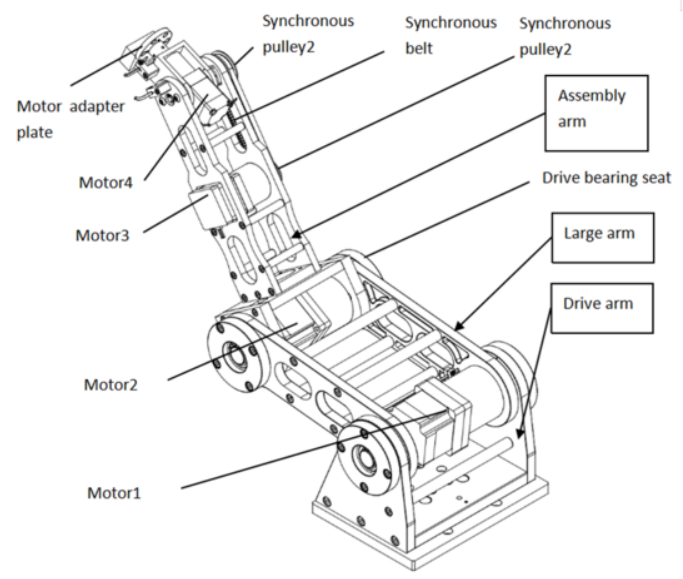

Figure 5. MDOF manipulator

The mechanical arm of the subject team is divided into four parts: drive arm, large arm, assembly arm and motor adapter plate (which can carry various detectors). It is a 6degree-of-freedom mechanical arm: drive arm and large arm rotate (drive by drive bearing seat), large arm and assembly arm rotate (drive by drive bearing seat), and the clamp plate of the motor on the assembly sub-arm rotates with step motor 3 (driven by synchronous pulley and synchronous belt), the adapter plate of motor 4 rotates (driven directly by motor 4 ), the arm moves in $\mathrm{X}$ direction and the arm rises and falls in $\mathrm{Z}$ direction. The structure of the drive bearing seat is shown in Figure 6 . The stepper motor is fixed on the side plate of the large arm. The motor shaft matches the hole of the arm drive shaft, the large arm drive shaft matches the deep groove ball bearing, the outer ring of the deep groove ball bearing matches the drive bearing seat, and the drive bearing seat is fixed on the clamp plate of the drive arm. Driving the bearing seat enables the stepper motor to rotate with the large arm while providing the drive force for the large arm to rotate.

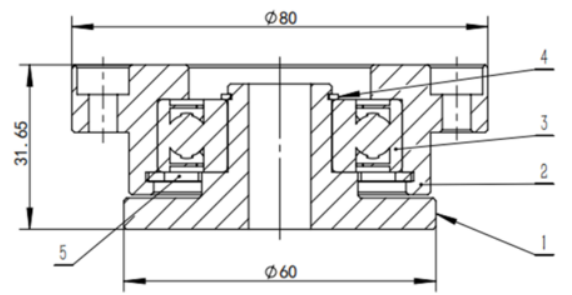

1-Drive shaft of large arm ; 2-Drive bearing seat; 3-Deep groove ball bearing; 4-Elastic retainer ring type A for 20-axis ; 5-Elastic retainer ring for holes Type A 48

Figure 6. Semi-sectional view of drive bearing seat 


\section{Manipulator Simulation}

As shown in Figure 7, the schematic diagram of the switch gear is shown. When detecting the ground wave, two detection points are set, and the detection points are set at the midpoint of $\mathrm{EF}$ and $\mathrm{GH}$ respectively.

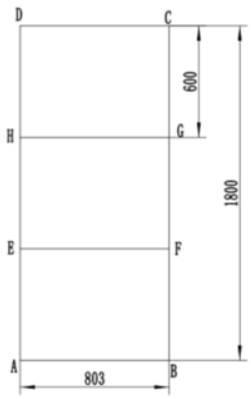

Figure 7. Schematic diagram of switch gear size

According to the requirements of the mechanical structure design and detection position accuracy of the switch gear live detection robot, it is necessary to simulate the manipulator on the robot, analyze the relationship between the pose of the end effector and the rotation angle of each joint, and use the D-H parameter method to study the kinematics of the manipulator [12]. As shown in Figure 2, the manipulator can be simplified as a combination of a four degree of freedom connecting rod and two sliding blocks (vertical and horizontal). Then the D-H parameter table of the manipulator is obtained according to the actual size, as shown in the Table 3.

Table 3. D-H parameter

\begin{tabular}{ccccc}
\hline Joint & $\theta$ & $\mathrm{d}$ & $\mathrm{a}$ & $\alpha$ \\
\hline $0-1$ & 0 & $\mathrm{~d}_{1}$ & 0 & $\frac{\pi}{2}$ \\
$1-2$ & 0 & $\mathrm{~d}_{2}$ & 0 & 0 \\
$2-3$ & $\theta_{1}$ & 0 & 0.206 & 0 \\
$3-4$ & $\theta_{2}$ & 0 & 0.270 & 0 \\
$4-5$ & $\theta_{3}$ & 0 & 0.122 & 0 \\
\hline
\end{tabular}

$\theta$-angle of rotation on z-axis; $d$-offset length of joint, $d_{1} \in[0,0.917], d_{2} \in[0,0.150]$; $a$ connecting rod length; $\alpha$-torsion angle, angle from $\mathrm{z}_{\mathrm{n}}$ to $\mathrm{z}_{\mathrm{n}+1}$.

The robotic toolbox in MATLAB is used to model the robot arm described in the table above after calculating the $\mathrm{D}-\mathrm{H}$ parameters, and the teaching mode generated by the teach function can be used to debug $\mathrm{Q}$ as shown in Figure 8 (q1, q2 equal $\mathrm{d}_{1}, \mathrm{~d}_{2}, \mathrm{q} 2$, $\mathrm{q} 3, \mathrm{q} 4$ are 1-2, 2-3, 3-4 joint rotation angles theta):

$\mathrm{Q}=[\mathrm{q} 1, \mathrm{q} 2, \mathrm{q} 3, \mathrm{q} 4, \mathrm{q} 5]$ 


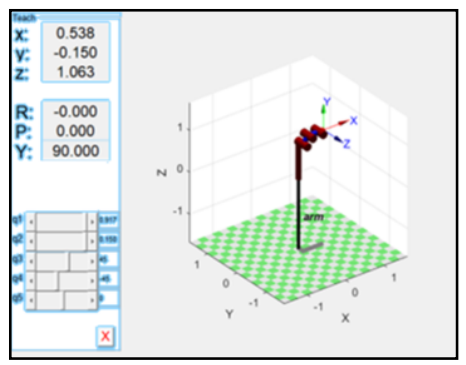

Figure 8. Manipulator simulation model

In order to verify that the manipulator can reach the specified position on the switch gear, the final position that the manipulator can reach needs to be depicted in the form of points. A series of random joint parameters $q$ are obtained by rand function, and then the forward kinematics solution function fkine of the toolbox is used to make $\mathrm{T}=\mathrm{fkine}$ (robot, $[\mathrm{q} 1(\mathrm{n}), \mathrm{q} 2(\mathrm{n}), \mathrm{q} 3(\mathrm{n}), \mathrm{q} 4(\mathrm{n}), \mathrm{q} 5(\mathrm{n})])$, to obtain the pose matrix $\mathrm{T}$ of the end effector,

$$
\mathrm{T}=\left[\begin{array}{l}
\mathrm{n}_{\mathrm{x}}, \mathrm{o}_{\mathrm{x}}, \mathrm{a}_{\mathrm{x}}, \mathrm{p}_{\mathrm{x}} \\
\mathrm{n}_{\mathrm{y}}, \mathrm{o}_{\mathrm{y}}, \mathrm{a}_{\mathrm{y}}, \mathrm{p}_{\mathrm{y}} \\
\mathrm{n}_{\mathrm{z}}, \mathrm{o}_{\mathrm{z}}, \mathrm{a}_{\mathrm{z}}, \mathrm{p}_{\mathrm{z}} \\
0,0,0,1
\end{array}\right]
$$

The position information of the end effector $\left(p_{x}, p_{y}, p_{z}\right)$ is consisted of the first three elements of the last column in the T matrix constitute. From this series of points, the reachable position in the manipulator space can be obtained. Since the robot does not move and the manipulator does not move in the y direction (horizontal sliding) during detection, the simulation result of $\mathrm{x}-\mathrm{z}$ plane is token, as shown in Figure 9:

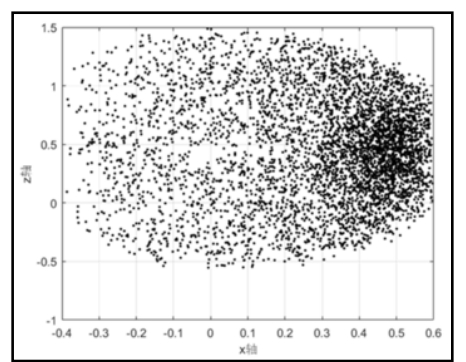

Figure 9. Point cloud distribution on x-z plane of manipulator position

The distribution of observation point cloud is dense in the range of $0.3-0.6 \mathrm{~m}$ in the $\mathrm{x}$-axis direction and $0.5-1.25 \mathrm{~m}$ in the $\mathrm{z}$-axis direction, which indicates that the probability of the manipulator reaching in this area is higher, so the detection point needs to be in this area as far as possible. Look at the two detection points set in Figure 7, whose z-axis coordinates are 0.6 and 1.2, which meet the z-axis region where the probability of the manipulator reaching.

In actual operation, the detecting robot cannot be affixed to the switch gear, so the end actuator is not vertical to the ground, but horizontal; and the detection of ground wave is only at the fixed position of the outer edge of the switch gear, so when the 
detecting robot detects ground wave, the manipulator does not move along the y-axis (where the $y$-axis is the y-axis of the simulation model, i.e. the horizontal sliding displacement of the manipulator is 0 ). Therefore, the initial and end positions of the arm are set as shown in Figure 10.

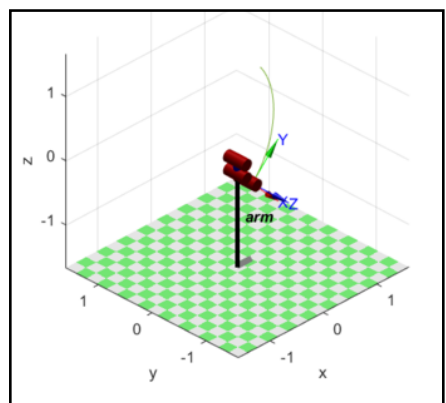

(a) Initial position

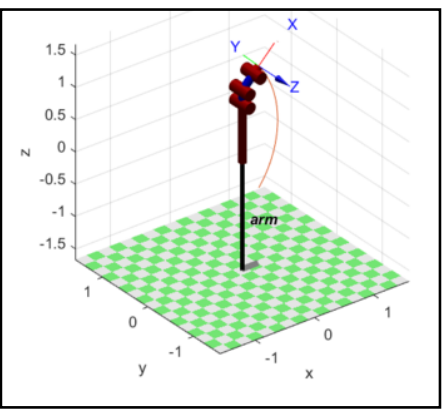

(b) End position

Figure 10. Initial and end positions of the manipulator

When trajectory simulation is carried out, the position and attitude matrix of initial state and final state are calculated first, then all joint parameters $q(n)$ between the two positions and attitudes are calculated by jtraj function in the toolbox. The $\mathrm{x}-\mathrm{z}$ plane simulation results are shown in Figure 11.

The x-range contains $(0.3,0.6)$ ranges, i.e., the end actuator of the arm is in the region where the approximate rate is reached when the initial and end positions are set, so the position requirements are met. In actual control, the initial and end positions of the arm can be set with reference to this article.

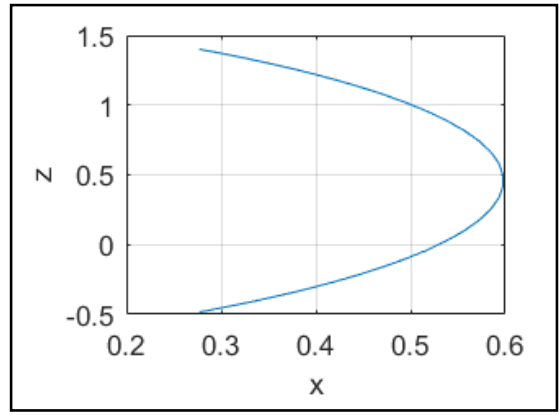

Figure 11. Plane trajectory of arm position $x-z$

\section{Summary}

Based on the dimensions of switch gear and switch gear room, this paper designs the structure of the switch gear live detection robot and carries out the selection calculation of the drive motor, which provides a reference for the manufacture of the switch gear live detection robot and has a certain preliminary theoretical contribution for reducing labor investment and automatic detection. Based on D-H parameter method, the simulation model of the manipulator and simulates the space position of the manipulator is established through MATLAB robotic toolbox, which provides a theoretical basis for 
setting the initial position and the end position of the actual control process. The setting can be selected by referring to the approximate rate position area of the cloud point map.

\section{References}

[1] Ren Shuangzan, Zeng Xiaoming, Wu Jingfeng, Yang Chuankai, Wu Hao and Li Hongjie. Development of Portable PD Detector for Switch gear Based on TEV and Ultrasound [J]. Electrical Measurements and Instruments, 2020, 57 (08): 135-139.

[2] Jiao Xia, Xiong Jun, Liang Zhongxi, Zhang Yu, Huang Xuexin, Zhang Guozhong, Zeng Yin. Detection and positioning of local discharge of 10kV switch gear [J]. Electrical Power Science and Engineering, 2019, 35 (11): 62-67.

[3] Chen Ming.On-line monitoring and fault diagnosis of high voltage switch gear [J]. Guangdong Science and Technology, 2013, 22 (16): 122+121.

[4] Zhang Bihui, Huang Weiwei, Xin Yichun, Guanshan. Research on High Voltage Switch gear Online Comprehensive Monitoring System Based on Internet of Things Information Integration [J]. Electronic Design Engineering, 2020, 28 (13): 101-107.

[5] Wang Yang, Su Bin, Zhao Hongbo. The concept and development trend of the power Internet of things [J]. Telecommunications Science, 2010, 26 (S3): 9-14.

[6] Wang Zhigao, Ye Youming, etc. Volume 5 Details of Transient Ground Voltage Partial Discharge Detection, General Management Regulations for Substation Detection of State Grid Corporation[DB/OL]. https://wenku.baidu.com/view/639e43165afb770bf78a6529647d27284a733752.html. 2020-3-23/2020$8-25$.

[7] Zhao Yuxin. Research on Cartesian Coordinate Robot System Based on Linear Motion Unit [D]. Qilu University of Technology, 2014:7.

[8] Zhao Yuxin, Shen Minde. Research status and development prospect of linear motion unit technology[J]. Journal of Shandong Light Industrial University (Natural Science Edition), 2013, 27 (04): 54-58.

[9] Xu Zhenwei. Design and Performance Analysis of Cartesian Handling Robot [D]. Shenyang University of Technology, 2017:24.

[10] Tao Zihang. Design and Research on Structure and Control System of Light Robot Arm [D]. University of Science and Technology of China, 2017:8.

[11] Li Xiaonan. Integral scheme of mechanical arm [J]. Metallurgy and Materials, 2019, 39 (01): 94-95+97.

[12] Zhou Tin. 4-DOF Joint Arm Motion Simulation [D]. Nanjing University of Aeronautics and Astronautics, 2015:17. 